\title{
A efetividade da acupuntura auricular no controle do estresse: Estudo experimental
}

\author{
The effectiveness of ear acupunture in stress control: An experimental study \\ La efectividad de la acupuntura del oído en el control del estrés: Un estudio experimental
}

Recebido: 14/04/2021 | Revisado: 23/04/2021 | Aceito: 27/04/2021 | Publicado: 11/05/2021

\author{
Carolina Neves Dias de Andrade \\ ORCID: https://orcid.org/0000-0002-8354-2619 \\ Universidade do Estado do Rio de Janeiro, Brasil \\ E-mail: carol.dias.andrade@gmail.com \\ Aline Rosa Fernandes Cardozo \\ ORCID: https://orcid.org/0000-0001-7406-9076 \\ Universidade do Estado do Rio de Janeiro, Brasil \\ E-mail: alinerosafc@gmail.com \\ Priscila Cristina da Silva Thiengo de Andrade \\ ORCID: https://orcid.org/0000-0003-0840-4838 \\ Universidade do Estado do Rio de Janeiro, Brasil \\ E-mail: profprithiengo@gmail.com \\ Ellen M. Peres \\ ORCID: https://orcid.org/0000-0003-4262-6987 \\ Universidade do Estado do Rio de Janeiro, Brasil \\ E-mail: ellenperes@globo.com \\ Helena Ferraz Gomes \\ ORCID: https://orcid.org/0000-0001-6089-6361 \\ Universidade do Estado do Rio de Janeiro, Brasil \\ E-mail: helenafg1@yahoo.com.br \\ Antonio Marcos Tosoli Gomes \\ ORCID: https://orcid.org/0000-0003-4235-9647 \\ Universidade do Estado do Rio de Janeiro, Brasil \\ E-mail: mtosoli@gmail.com \\ Carolina Cabral Pereira da Costa \\ ORCID: https://orcid.org/0000-0002-0365-7580 \\ Universidade do Estado do Rio de Janeiro, Brasil \\ E-mail carolcuerj@hotmail.com \\ Lívia Fajin de Mello dos Santos \\ ORCID: https://orcid.org/0000-0002-5613-7976 \\ Universidade do Estado do Rio de Janeiro, Brasil \\ E-mail: liviafajin@gmail.com \\ Alessandra Sant'anna Nunes \\ ORCID: https://orcid.org/0000-0001-7435-2568 \\ Universidade do Estado do Rio de Janeiro, Brasil \\ E-mail: asantnunes@yahoo.com.br \\ Bruna Maiara Ferreira Barreto Pires \\ ORCID: https://orcid.org/0000-0002-5584-8194 \\ Universidade Federal Fluminense, Brasil \\ E-mail: bruna.barreto07@gmail.com
}

\begin{abstract}
Resumo
Objetivo: avaliar a efetividade da acupuntura auricular em estudantes de graduação com níveis variados de estresse. Materiais e métodos: Trata-se de um estudo quantitativo de natureza experimental, controlado, com dois grupos: intervenção e controle. Para definir a amostra dos sujeitos participantes foi utilizado o Inventário de Sinais e Sintomas de Stress de Lipp - ISSL, em seguida aqueles que obtiveram estresse positivo fizeram uma sessão semanal de 15 minutos à 1 hora durante quatro semanas. Para o grupo intervenção os pontos escolhidos foram: Shen Men, Fígado, Pulmão/Coração, Rim e ansiedade. O grupo controle teve os seus pontos escolhidos de acordo com as queixas apontadas. Após a última sessão os estudantes responderam o ISSL. Resultados: Participaram do estudo 27 estudantes e foi encontrada relação significativa entre sexo, idade, tempo de deslocamento para a faculdade, número de transportes e participação em projetos extracurriculares. Estes foram divididos em grupo intervenção (13) e grupo controle (14). Após a acupuntura auricular, no grupo intervenção, foi obtido um aumento de 30,7\% de estudantes sem estresse e no grupo controle, esse aumento foi de 64,3\%, aproximadamente duas vezes maior que no primeiro. Considerações finais: Permite-se concluir que as taxas de estresse dos participantes estão próximas às encontradas em outros estudos, e que a acupuntura auricular demostrou ser uma técnica que pode auxiliar na redução dos sinais e sintomas de estresse nesta população.
\end{abstract}

Palavras-chave: Auriculoterapia; Estresse; Graduação. 


\begin{abstract}
Objective: to evaluate the effectiveness of ear acupuncture in undergraduate students with varying levels of stress. Materials and methods: This is a quantitative study of an experimental, controlled nature, with two groups: intervention and control. To define the sample of the participants, the Lipp Stress Signs and Symptoms Inventory ISSL was used, followed by those who obtained positive stress had a weekly session of 15 minutes at 1 hour for four weeks. For the intervention group, the points chosen were: Shen Men, Liver, Lung/Heart, Kidney and anxiety. The control group had its points chosen according to the complaints pointed out. After the last session the students responded to the ISSL. Results: Twenty-seven students participated in the study and a significant relationship was found between gender, age, time of travel to college, number of transportation and participation in extracurricular projects. These were divided into intervention group (13) and control group (14). After ear acupuncture, in the intervention group, an increase of $30.7 \%$ of students without stress was obtained and in the control group, this increase was $64.3 \%$, approximately twice as high as in the first. Final considerations: It is possible to conclude that the stress rates of the participants are close to those found in other studies, and that ear acupuncture proved to be a technique that can help reduce the signs and symptoms of stress in this population.
\end{abstract}

Keywords: Auriculotherapy; Stress; Graduation.

\title{
Resumen
}

Objetivo: evaluar la efectividad de la acupuntura del oído en estudiantes de pregrado con diferentes niveles de estrés. Materiales y métodos: Se trata de un estudio cuantitativo de carácter experimental y controlado, con dos grupos: intervención y control. Para definir la muestra de los participantes, se utilizó el Inventario de Signos de Estrés y Síntomas de Lipp - ISSL, seguido por aquellos que obtuvieron estrés positivo tuvieron una sesión semanal de 15 minutos a 1 hora durante cuatro semanas. Para el grupo de intervención, los puntos elegidos fueron: Shen Men, Hígado, Pulmón/Corazón, Riñón y Ansiedad. El grupo de control tenía sus puntos elegidos de acuerdo con las quejas señaladas. Después de la última sesión, los estudiantes respondieron a la ISSL. Resultados: Veintisiete estudiantes participaron en el estudio y se encontró una relación significativa entre género, edad, tiempo de viaje a la universidad, número de transporte y participación en proyectos extracurriculares. Estos se dividieron en grupo de intervención (13) y grupo de control (14). Después de la acupuntura del oído, en el grupo de intervención, se obtuvo un aumento del $30,7 \%$ de los estudiantes sin estrés y en el grupo de control, este aumento fue del 64,3\%, aproximadamente el doble que en el primero. Consideraciones finales: Es posible concluir que las tasas de estrés de los participantes están cerca de las que se encuentran en otros estudios, y que la acupuntura del oído demostró ser una técnica que puede ayudar a reducir los signos y síntomas del estrés en esta población.

Palabras clave: Auriculoterapia; Estrés; Graduación.

\section{Introdução}

Atualmente a população vive imersa em um cenário imediatista e com considerável aceleração do ritmo de vida. Há uma correlação direta à necessidade de atender demandas de produtividade acadêmicas, profissionais ou sociais que geram consequências negativas no âmbito biopsicossocial de cada indivíduo. A partir disso, surgem cada vez mais frequentemente problemas como o estresse.

O estresse é uma reação fisiológica vivenciada por pessoas que estão lidando com situações conflitantes em diversos contextos internos e externos que implicam a necessidade de uma adaptação para enfrentá-los, no entanto, a pessoa precisa transpor seus limites para lidar com elas. Se os níveis de estresse persistem a adaptabilidade inicial pode dar lugar a alterações fisiológicas, emocionais, endócrinas, imunológicas e neurológicas patológicas, tornando o indivíduo vulnerável ao adoecimento (Kestemberg, Rosa, da Silva, Fabri, \& Regazzi, 2017).

Os estudantes que ingressam no ensino superior geralmente estão em uma transição de cobranças acadêmicas mais flexíveis advindas do ensino médio. A faculdade traz novas responsabilidades e perspectivas que muitas vezes refletem em situações que o estudante não está preparado para encarar, sendo sua adaptação um processo lento. Se o estudante não tiver oportunidade de expressar seus sentimentos à colegas ou professores sua tendência é ocultar o sentimento o que pode desencadear outros problemas além do estresse (Graner \& Cerqueira, 2019).

Desta forma, cuidar dos estudantes de graduação torna-se imprescindível para o processo de humanização em saúde. Estudantes saudáveis melhoram o rendimento e lidam melhor com as situações de conflito diárias. A busca por intervenções e formas de controle do estresse deve ser constante e a acupuntura auricular pode ser uma proposta promissora para este cuidado. 
A acupuntura auricular é um tratamento advindo da Medicina Tradicional Chinesa (MTC) que data 4.000 anos de buscas e validações com base na experiência. Ela é na atualidade uma das mais populares dentro e fora da China, por tratar-se, principalmente, de um método não invasivo. Essa técnica terapêutica consiste em um método de diagnóstico e de tratamento de desordens físicas e psicossomáticas por meio da estimulação de áreas específicas no pavilhão auricular e costuma ser utilizada de forma coadjuvante a outras como a acupuntura sistêmica, a fitoterapia, a massagem, os exercícios físicos, a dieta, a moxabustão, ventosa entre outras (Moura et al., 2019; Do Prado et al., 2012). A acupuntura auricular é reconhecida mundialmente e no Brasil foi incluída na Política nacional de Práticas Integrativas e Complementares do Sistema Único de Saúde (SUS) implementada pela Portaria Nº 97, de 03 de maio de 2006 (Ravaglio, Silveira \& Blev, 2018).

Assim sendo, considerando a promoção da saúde dos estudantes e a relevância da temática, levanta-se a hipótese de que a intervenção com acupuntura auricular seja eficaz no controle do estresse. Nesse contexto, o estudo tem como objetivo avaliar a efetividade da acupuntura auricular em estudantes de graduação com níveis variados de estresse. Este estudo é relevante no âmbito do cuidado, à medida que pode servir de modelo de intervenção para outras instituições interessadas em trabalhar a diminuição do estresse em graduandos.

\section{Metodologia}

Estudo quantitativo de natureza experimental, controlado, com dois grupos: intervenção e controle. A pesquisa experimental de forma objetiva consiste em determinar um objeto de estudo, selecionar as variáveis capazes de influenciar o objeto e definir as formas de controle e de observação dos efeitos que a variável produz no objeto (Silva Dutra \& Nunes dos Reis, 2016). Também se caracteriza como pesquisa de cunho quantitativo tendo em vista que busca quantificar as relações entre as variáveis adotando estratégia sistemática, objetiva e rigorosa para gerar e refinar o conhecimento (Sousa, Driessnack, \& Mendes, 2007).

Os participantes do estudo foram graduandos de uma universidade pública, no estado do Rio de Janeiro e a seleção da amostra caracterizou-se como não probabilística, contando com indivíduos prontamente disponíveis. Para defini-la, foi utilizado o Inventário de Sintomas de Stress para Adultos - ISSL, que contém 53 questões fechadas, dentre elas 37 somáticas (avalia sintomas físicos) e 19 psicológicas (avalia sintomas psicológicos) que permitem avaliar se as pessoas tem ou não estresse e em que fase está (alerta, resistência, quase-exaustão ou exaustão). O questionário é dividido em três partes, os indivíduos preenchem as opções de sintomas que sentiram nas últimas 24 horas, na última semana e no último mês (Lipp, 2000).

Após a aplicação do questionário, entraram para o grupo intervenção ou controle, de forma aleatória, aqueles que obtiveram estresse positivo (fases de resistência, quase exaustão e exaustão) ou estavam sem estresse. E fizeram uma sessão por semana durante quatro semanas, respondendo novamente ao LIPP uma semana após a última sessão.

O instrumento foi aplicado a 40 voluntários que foram esclarecidos sobre a acupuntura auricular, os objetivos da pesquisa e após, todos realizaram a assinatura do Termo de Consentimento Livre e Esclarecido (TCLE). Foram excluídos apenas aqueles que tiveram a perda de seguimento das sessões, por desistência (quatro), sendo a amostra final constituída por 27 estudantes: 13 do grupo intervenção e 14 do grupo controle. A pesquisa foi realizada no horizonte temporal de outubro de 2019 a janeiro de 2021.

As sessões duraram de 15 minutos à 1 hora. Para o grupo intervenção os pontos escolhidos foram: Shen Men, por ser um ponto sedante, Fígado por regular o fluxo de energia corporal e diminuir a dor, Pulmão/Coração, Rim e ansiedade como mostrado na Figura 1. Já o grupo controle teve os seus pontos escolhidos de acordo com as queixas apontadas. Na primeira sessão foi aplicado o LIPP, em seguida foi realizada uma massagem em ambas as orelhas; após a devida localização dos pontos 
reativos, foi feita a higienização com algodão e álcool etílico $70 \%$ do pavilhão auricular e aplicação das sementes de mostarda afixadas com micropore ou esparadrapo, permanecendo por sete dias.

Figura 1 - Pontos auriculares do Grupo Intervenção

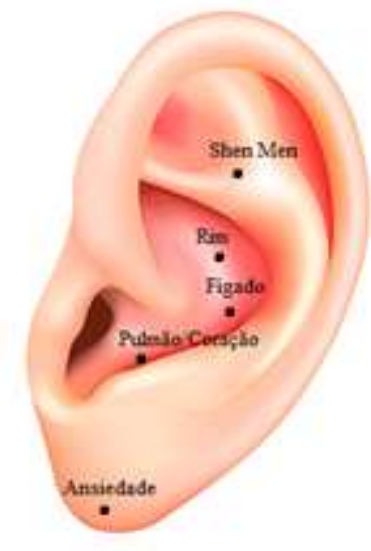

Fonte da imagem: https://bitlybr.com/4hSO4oc.

Os dados foram analisados através de técnicas pertencentes à estatística descritiva simples, e foram realizados cálculos estatísticos para estimar os parâmetros. Os resultados de variáveis quantitativas foram descritas por médias, medianas, valores mínimos, máximos e desvios padrões. Variáveis categóricas foram descritas por frequências e percentuais.

Este estudo faz parte da pesquisa "Sistematização da Assistência de Enfermagem na perspectiva de inovação tecnológica nas Unidades de Saúde da UERJ: Pesquisa Clínica”, cujo CAAE: 16427419.3.0000.5259 e parecer nº 3.443.800, em 08 de julho de 2019.

\section{Resultados e Discussão}

\subsection{Caracterização dos participantes do estudo}

Grupo intervenção: participaram treze estudantes: dois homens (15,4\%) e 11 mulheres $(84,6 \%)$; um participante possui idade entre 18 a 20 anos (7,7\%), oito entre 21 a 23 anos (61,5\%) e quatro entre 24 a 26 anos (30,8\%). A idade média dos participantes foi de 23,5 anos, com desvio padrão de 2,7, e variou de 18 a 34 anos. Dois eram cotistas e bolsistas de projetos $(15,3 \%)$, um era cotista e voluntário em projeto (7,7\%), dois eram apenas bolsistas (15,3\%), dois eram apenas voluntários (15,3\%). Cinco não eram nem cotistas nem participantes de projetos, seja como voluntário seja como bolsista $(38,5 \%)$. Do grupo, três trabalhavam (23\%). Um estudante relatou ter filho (7,7\%). A média de tempo de deslocamento para a faculdade foi de 36,30 min, com desvio padrão de 54, variando de $2 \mathrm{~min}$ a $180 \mathrm{~min}$ ( 3 horas). A média de transportes usados para chegar à faculdade foi de dois, variando de 0 (a pé) a dois transportes.

Grupo controle: participaram quatorze estudantes: três homens (21,4\%) e 11 mulheres (78,6\%). Dois participantes entre 18 a 20 anos (14,3\%), oito entre 21 a 23 anos (57,1\%), três entre 24 a 26 anos (21,4\%) e 1 entre 27 a 34 anos (7,2\%). A idade média dos participantes do grupo controle foi de 23,4 anos, com desvio padrão de 3,7, e variou de 18 a 34 anos. Dos 14 estudantes, 6 eram cotistas (42,8\%), 8 não eram cotistas (57,2\%), 4 eram cotistas e bolsistas de projetos (28,6\%), 2 eram cotistas e voluntários em projeto (14,3\%), 4 eram apenas bolsistas (28,6\%), 4 eram apenas voluntários (28,6\%) e não houveram estudantes que não fossem nem cotistas nem participantes de projetos, seja como voluntário seja como bolsista. Dos 14 
estudantes, 3 trabalhavam $(21,4 \%)$ e nenhum estudante tem filho. A média de tempo de deslocamento para a faculdade foi de $60 \mathrm{~min}$, com desvio padrão de 39,2, variando de $20 \mathrm{~min}$ a $120 \mathrm{~min}$ (2 horas). A média de transportes usados para chegar à faculdade foi de 1,4 variando de 1 a 2 transportes.

Conforme descrito, a maioria dos estudantes que participaram do estudo eram do sexo feminino (22, 81,5\%), com idade entre 18 a 34 anos (27,100\%). Em 2018, a Associação Nacional dos Dirigentes das Instituições de Ensino Superior (Andifes, 2018), realizou um levantamento do perfil socioeconômico dos estudantes de graduação das universidades federais do Brasil. De acordo com esse levantamento, 54,6\% dos estudantes universitários são do sexo feminino, a média de idade está entre 24 e 25 anos $(49,3 \%)$.

Estes números podem ser compreendidos pois as mulheres são a maioria absoluta no ensino médio devido à evasão dos estudantes do sexo masculino que têm, por sua vez, entrada "precoce" no mercado de trabalho. Outro estudo apontou a maior prevalência do estresse em acadêmicas, isso estaria relacionado às responsabilidades com as atividades obrigatórias da universidade, trabalho e cuidados com o lar e a família. A sobrecarga de responsabilidades aumenta a produção de hormônios, como o cortisol e a adrenalina, que atingem duas mulheres para cada homem, implicando altos níveis de exaustão emocional e aparecimento de quadros de ansiedade, pânico e depressão (Cestari, Barbosa, Florêncio, Pessoa \& Moreira, 2017)

No que se referiu à faixa etária associada ao estresse, muitos estudos referem o predomínio de estudantes na faixa etária entre 20 e 25 anos, o que pode ser percebido pela população desse estudo. Esse dado pode relacionar-se à possibilidade de os estudantes, nessa faixa etária, sentirem-se inseguros quanto às exigências da formação profissional e em relação à profissão escolhida. Além disso, durante o período de formação profissional, o aluno se depara com um novo ambiente, muitas vezes, diferente e distante de seu contexto de vida e a necessidade de adaptação às novas exigências e obrigações escolares, o que pode contribuir para elevar o nível de estresse nesses discentes (Bublitz, Guido, Lopes \& Freitas, 2016).

Verificou-se a associação entre o estresse, o tempo de deslocamento e o número de transportes necessários para chegar à faculdade. Um estudo realizado por Hirsch et al. (2018) refere que o tempo de deslocamento entre a moradia do estudante e a faculdade juntamente com o transporte público utilizado podem ser considerados fatores estressantes para o cotidiano do acadêmico. O transporte público é fundamental e o serviço prestado por ele influencia diretamente a qualidade de vida das pessoas. Assim, a facilidade até o local de embarque, ônibus e metrô com superlotação, o tempo de espera, o deslocamento até o destino final, as condições do veículo, a segurança (possíveis acidentes e roubos), a disponibilidade de informações acerca do transporte e o comportamento dos prestadores de serviço são os principais fatores que colaboram para a falta de qualidade de vida o que inclui o estresse cotidiano gerado pelo transporte público (Hirsch et al., 2018).

Verificou-se a associação entre o estresse e a participação em projetos de extensão ou de pesquisa com e sem bolsa auxílio. Em um estudo, foi observado que os alunos que estavam na instituição com dedicação exclusiva, ou seja, que não trabalhavam, eram, cada vez mais, instigados a participarem de grupos de pesquisa, projetos de extensão, monitoria, eventos e realização de cursos de atualização, o que pode ter influenciado na avaliação do estresse desses discentes. (Bublitz, Guido, Lopes \& Freitas, 2016).

Alguns pesquisadores destacam que algumas características sociodemográficas e acadêmicas dos discentes podem influenciar em seus níveis de estresse (Bublitz, Guido, Lopes \& Freitas, 2016). No entanto, nesse estudo, não foram encontradas diferenças estatísticas significativas ou não foram obtidos dados suficientes para alcançar um padrão na relação entre período de estudo, trabalho fora, filhos e o nível de estresse dos estudantes que participaram do estudo.

Com relação ao curso superior, 92,6\% dos participantes cursavam graduação na área da saúde e 7,4\% cursavam outras áreas. Entre a população universitária, embora cada área tenha seu fator estressor associado, existe um grupo de risco para o desenvolvimento do estresse: os estudantes da área da saúde. Esse grupo está refém do estresse por ter que lidar com novas adaptações, novas preocupações, ritmo de estudo diferenciado e afastamento do ambiente familiar, necessidade de desenvolver 
responsabilidade emocional acentuada como ferramenta de trabalho, além de se depararem com questões de difícil compreensão no âmbito da saúde e da doença como a morte e o sofrimento alheio, onde acabam por perceber as limitações da sua atuação (Pacheco, Vilela \& Carlos, 2013).

\subsection{Efetividade da acupuntura auricular}

A Tabela 1 apresenta a distribuição dos níveis por fase de estresse observado nos pacientes antes e após a aplicação da acupuntura auricular, divididos em grupo intervenção e grupo controle. Observa-se: estudantes sem estresse (SE); préintervenção: Intervenção $(n=2,15,3 \%)$, Controle $(n=3,21,4 \%)$; e após intervenção: Intervenção $(n=6,46 \%)$, Controle $(n=12$, $85,7 \%)$.

Tabela 1- Distribuição dos níveis de estresse por fase antes e após as sessões. Rio de Janeiro, 2021.

\begin{tabular}{|c|c|c|c|c|c|c|c|c|c|c|c|}
\hline \multicolumn{6}{|c|}{ Grupo Intervenção } & \multicolumn{6}{|c|}{ Grupo Controle } \\
\hline \multicolumn{3}{|c|}{ Pré Intervenção } & \multicolumn{3}{|c|}{ Pós Intervenção } & \multicolumn{3}{|c|}{ Pré Intervençãa } & \multicolumn{3}{|c|}{ Pós Intervenção } \\
\hline $\begin{array}{l}\text { Nível } \\
\text { estresse }\end{array}$ & $\begin{array}{l}\text { ISSL1 } \\
\text { (n) }\end{array}$ & $\%$ & $\begin{array}{l}\text { Nível } \\
\text { estresse }\end{array}$ & $\begin{array}{l}\text { ISSL1 } \\
\text { (n) }\end{array}$ & & $\begin{array}{l}\text { Nível } \\
\text { estres } \\
\text { se }\end{array}$ & $\begin{array}{l}\text { ISSL1 } \\
\text { (n) }\end{array}$ & & $\begin{array}{l}\text { Nível } \\
\text { estres } \\
\text { se }\end{array}$ & $\begin{array}{l}\text { ISSL1 } \\
\text { (n) }\end{array}$ & \\
\hline SE & 2 & 15,3 & SE & 6 & 46 & SE & 3 & 21,4 & SE & 12 & 85,7 \\
\hline FA & 0 & 0 & FA & 0 & 0 & FA & 0 & 0 & FA & 0 & 0 \\
\hline FR & 7 & 54 & FR & 7 & 54 & FR & 9 & 64,3 & FR & 2 & 14,3 \\
\hline $\mathrm{QE}$ & 3 & 23 & $\mathrm{QE}$ & 0 & 0 & $\mathrm{QE}$ & 2 & 14,3 & $\mathrm{QE}$ & 0 & 0 \\
\hline FE & 1 & 7,7 & $\mathrm{FE}$ & 0 & 0 & $\mathrm{FE}$ & 0 & 0 & $\mathrm{FE}$ & 0 & 0 \\
\hline Total & 13 & 100 & Total & 13 & 100 & Total & 14 & 100 & Total & 14 & 100 \\
\hline
\end{tabular}

Legenda: SE- sem estresse, FA- fase de alerta, FR- fase de resistência, QE- quase exaustão, FE- fase de exaustão. Fonte: Autores (2021).

Pode-se observar nos dados referentes ao grupo intervenção que dos dois estudantes inicialmente sem estresse, um manteve-se sem estresse e outro entrou na fase de resistência, tendo assim, desenvolvido estresse. Dos 11 estudantes restantes desse grupo, cinco saíram das fases de resistência, quase exaustão e exaustão para SE, enquanto seis estudantes mantiveram-se na mesma fase, embora com sintomas reduzidos.

Em relação ao grupo controle, três estudantes iniciaram a terapia sem estresse e mantiveram-se sem estresse ao final da mesma. Dois estudantes iniciaram a terapia na fase de resistência e mantiveram-se na fase de resistência, embora com menos sintomas. Nove estudantes iniciaram a terapia nas fases de resistência e quase-exaustão e após a intervenção evoluíram para sem estresse.

Não foram obtidos estudantes na fase de alerta (FA) em nenhum dos grupos.

Em relação à fase de resistência (FR), pré intervenção: Intervenção $(n=7,54 \%)$; Controle $(n=9,64,3 \%)$; e após intervenção: Intervenção ( $\mathrm{n}=7,54 \%)$; Controle ( $\mathrm{n}=2,14,3 \%)$.

Em relação ao grupo intervenção sete estudantes iniciaram a terapia na fase de resistência, após a intervenção, dois evoluíram para sem estresse, cinco mantiveram-se nessa fase, um evoluiu da fase de quase exaustão para a fase de resistência e um estudante sem estresse passou a desenvolvê-lo entrando na fase de resistência.

Referente ao grupo controle, nove estudantes iniciaram na fase de resistência e sete evoluíram para sem estresse enquanto dois mantiveram-se nessa fase, embora com menos sintomas. 
Em relação a fase de quase exaustão (QE), na pré intervenção: Intervenção (n=3, 23\%); Controle (n=2, 14,3\%) e após intervenção com a acupuntura auricular, no grupo Intervenção dois evoluíram para sem estresse e um entrou na fase de resistência. No grupo Controle, os dois evoluíram para sem estresse.

E por fim, apenas um participante $(7,7 \%)$ que se encontrava na fase de exaustão (FE), pertencia ao grupo Intervenção $(\mathrm{n}=1,7,7 \%)$ e após a auriculoterapia, foi classificado como sem estresse. Após a intervenção, nenhum participante se manteve na condição de quase exaustão e exaustão.

Em relação ao grupo intervenção, três estudantes iniciaram a terapia na fase de quase exaustão e, após a intervenção, dois evoluíram para sem estresse e um evoluiu para a fase de resistência. O estudante desse grupo que iniciou a intervenção na fase de exaustão, evoluiu para sem estresse após a terapia.

Referente ao grupo controle, dois estudantes iniciaram a terapia na fase de quase exaustão e ambos evoluíram para sem estresse. Não houveram estudantes que iniciaram a terapia na fase de exaustão e nenhum entrou nessa fase após a intervenção.

Dos participantes que apresentaram algum nível de estresse, aqueles com predomínio de sintomas psicológicos: Intervenção ( $\mathrm{n}=11,100 \%)$; Controle $(\mathrm{n}=10,90,9 \%)$ e sintomas físicos presente apenas no grupo Controle $(\mathrm{n}=1,9,1 \%)$. Após a intervenção, mantiveram sintomas psicológicos: Intervenção $(n=7,63,6 \%)$; Controle $(n=2,18,2 \%)$ e nenhum participante desenvolveu ou manteve sintomas físicos.

A partir da análise dos dados obtidos foi possível perceber que os resultados encontrados no grupo controle foram superiores àqueles encontrados no grupo intervenção. Enquanto no grupo intervenção foi obtido um aumento de 30,7\% nos estudantes sem estresse após intervenção, no grupo controle esse aumento foi de 64,3\%, duas vezes maior para aqueles tratados sem limitação de pontos auriculares. Quando se trata de uma pesquisa científica nos moldes dos ensaios clínicos controlados e randomizados, a realidade do tratamento em MTC parece conflitar com o que preconizamos ser fundamental, que seria o uso de protocolos fechados. (De Andrade, et al., 2020)

\section{Considerações Finais}

Foi demonstrado nesta pesquisa que os participantes deste estudo têm um nível de estresse próximo ao encontrado na literatura. Uma pesquisa recente, realizada em São Paulo, buscou identificar a presença de estresse e depressão entre 88 estudantes do último ano da graduação de cursos na área da saúde, neste, aplicou-se a Escala de Stress Percebido na população, constatando que 73,9\% dos discentes apresentaram posição média de estresse. (Cestari, Barbosa, Florêncio, Pessoa \& Moreira, 2017). Os resultados do presente estudo permitem concluir que o nível de estresse dos participantes é médio, bem como os resultados encontrados em outros estudos, estando sua maioria na fase de resistência. Foram observados casos de estresse alto na fase de quase exaustão e exaustão entre os indivíduos. Diante do exposto, medidas que busquem auxiliar os graduandos a lidar com o estresse universitário tornam-se uma questão de humanização. Estratégias de enfrentamento aos estressores emocionais devem fazer parte da rotina diária destes estudantes.

Nos achados do presente estudo observou-se que o uso do protocolo aberto, ou seja, sem limitação de pontos auriculares e levando em consideração o tratamento individualizado apresentou melhores resultados em detrimento do protocolo fechado. Dois estudos encontraram resultados semelhantes à pesquisa em tela, demonstrando que a auriculoterapia sem protocolo, ou seja, levando em consideração as queixas levantadas pelo paciente apresentou melhor resultado, comparativamente, àquele que utilizou uma combinação específica de pontos (ou protocolo), quanto ao tamanho de efeito e aos percentuais de mudança, demonstrando que a auriculoterapia individualizada amplia o alcance da técnica (kurebayashi \& Silva, 2014; Kurebayashi \& Silva, 2015) 
Como inferido neste estudo, a acupuntura auricular pode ser um complemento para o controle de estresse, pois demonstrou eficácia na sua redução. Com o benefício de tratar-se de uma técnica de baixo custo, não invasiva, de fácil aplicação e com poucas contraindicações, podendo ser realizada no próprio ambiente universitário, com o mínimo de prejuízo ao andamento de sua rotina.

Outra questão levantada por este estudo implica que devido ao tamanho da amostra, não fica claro se o número de sessões de auriculoterapia a que os participantes foram submetidos influenciou na melhora do quadro de estresse, o que pode indicar fatores individuais associados a este quadro que não foram avaliados neste estudo.

Devido às limitações desta pesquisa, estudos posteriores se fazem necessários, com uma amostra maior e um tratamento mais longo. Outra questão que pode ser abordada posteriormente são as variáveis que influenciam o estresse, pois se trata de um processo multifatorial, a fim de verificar quais os benefícios do tratamento com auriculoterapia em longo prazo, além de investigar a relação entre o número de sessões e a melhora do quadro de estresse.

\section{Referências}

Associação Nacional dos Dirigentes das Instituições de Ensino Superior (2018). V Pesquisa Nacional de Perfil Socioeconômico e Cultural dos (as) Graduandos (as) das IFES. Brasília, 2019

Bublitz, S., Guido, L. D. A., Lopes, L. F. D., \& Freitas, E. D. O. (2016). Associação entre estresse e características sociodemográficas e acadêmicas de estudantes de enfermagem. Texto \& Contexto-Enfermagem, 25(4), e2440015. doi: 10.1590/0104-07072016002440015

Cestari, V. R. F., Barbosa, I. V., Florêncio, R. S., Pessoa, V. L. M. D. P., \& Moreira, T. M. M. (2017). Estresse em estudantes de enfermagem: estudo sobre vulnerabilidades sociodemográficas e acadêmicas. Acta Paulista de Enfermagem, 30(2), 190-196. doi: 10.1590/1982-0194201700029

De Andrade, P. C. S. T., de Andrade, C. N. D., Fernandes, L. F. G., Mouta, R. J. O., Risi, L. R., \& Souto, B. R. (2020). A auriculoterapia no controle do estresse da equipe de enfermagem: revisão integrativa da literatura. Research, Society and Development, 9(9), e911998307. doi: 10.33448/rsd-v9i9.8307

Prado, J. M., Kurebayashi, L. F. S., \& da Silva, M. J. P. (2012). Eficácia da auriculoterapia para diminuição de estresse em estudantes de enfermagem: ensaio clínico randomizado. Revista Latino-Americana de Enfermagem, 20(4), 727-735. doi: 10.1590/0104-1169.3239.2426

Graner, K. M., \& Cerqueira, A. T. D. A. R. (2019). Revisão integrativa: sofrimento psíquico em estudantes universitários e fatores associados. Ciência \& Saúde Coletiva, 24(4), 1327-1346. doi: 10.1590/1413-81232018244.09692017

Hirsch, C. D., Barlem, E. L. D., Almeida, L. K. D., Tomaschewski-Barlem, J. G., Lunardi, V. L., \& Ramos, A. M. (2018). Fatores percebidos pelos acadêmicos de enfermagem como desencadeadores do estresse no ambiente formativo. Texto \& Contexto-Enfermagem, 27(1), e0370014. doi: 10.1590/010407072018000370014

Hirsch, C. D., Barlem, E. L. D., Tomaschewski-Barlem, J. G., Lunardi, V. L., \& Oliveira, A. C. C. D. (2015). Predictors of stress and coping strategies adopted by nursing students. Acta Paulista de Enfermagem, 28(3), 224-229. doi: 10.1590/1982-0194201500038

Kestenberg, C. C. F., Rosa, B. M. S., da Silva, A. V., Fabri, J. M. G., \& Regazzi, I. C. R. (2017). Estresse em graduandos de enfermagem. Revista Enfermagem UERJ, 25, e26716. doi: 10.12957/reuerj.2017.26716

Kurebayashi, L. F. S., \& Silva, M. J. P. D. (2014). Eficácia da auriculoterapia chinesa para o estresse em equipe de enfermagem: ensaio clínico randomizado. Revista Latino-Americana de Enfermagem, 22(3), 371-378. doi: 10.1590/0104-1169.3239.2426

Kurebayashi, L. F. S., \& Silva, M. J. P. D. (2015). Auriculoterapia Chinesa para melhoria de qualidade de vida de equipe de Enfermagem. Revista Brasileira de Enfermagem, 68(1), 117-123. doi: 10.1590/0034-7167.2015680116

Lipp, M. E. N. (2000). Manual do inventário de sintomas de stress para adultos de Lipp (ISSL). São Paulo: Casa do Psicólogo

Moura, C. D. C., Chaves, E. D. C. L., Cardoso, A. C. L. R., Nogueira, D. A., Azevedo, C., \& Chianca, T. C. M. (2019). Acupuntura auricular para dor crônica nas costas em adultos: revisão sistemática e metanálise. Revista da Escola de Enfermagem da USP, 53, e03461. doi: 10.1590/s1980-220x2018021703461

Pacheco, A. E., Vilela, S. C., \& Carlos, A. L. S. (2013). Síndrome de burnout e estresse em graduandos de enfermagem. Revista de Enfermagem do CentroOeste Mineiro, 3(3), 780-787. doi: 10.19175/recom.v0i0.415

Ravaglio, A. V. M., Silveira, L. R. V., \& Blev, A. L. (2018). A influência da auriculoterapia nos níveis de estresse de profissionais de enfermagem de UTI pediátrica. Rev Bras Terap Saúde, 9(1), 1-7. doi: 10.7436/rbts-2018.09.01.01

Silva Dutra, H., \& Nunes dos Reis, V. (2016). Desenhos de estudos experimentais e quase-experimentais: definições e desafios na pesquisa em enfermagem. Revista de Enfermagem UFPE, 10(6), 2230-41. doi: 10.5205/1981-8963-v10i6a11238p2230-2241-2016

Sousa, V. D., Driessnack, M., \& Mendes, I. A. C. (2007). Revisão dos desenhos de pesquisa relevantes para enfermagem. Parte 1: desenhos de pesquisa quantitativa. Rev Latino-am Enfermagem, 15(3), 502-507. doi: 10.1590/S0104-11692007000300022 